J. Klin. Endokrinol. Stoffw. 2020 · 13:119-123 https://doi.org/10.1007/s41969-020-00109-6 Online publiziert: 2 . September 2020 (c) Der/die Autor(en) 2020

\section{Christian Trummer · Verena Theiler-Schwetz · Stefan Pilz}

Klinische Abteilung für Endokrinologie und Diabetologie, Univ.-Klinik für Innere Medizin, Medizinische Universität Graz, Graz, Österreich

\title{
Amiodaron-induzierte Thyreopathien
}

\section{Einleitung}

Amiodaron ist ein Klasse III-Antiarrhythmikum, das zur Prophylaxe und zur Behandlung von kardialen Arrhythmien eingesetzt wird. Trotz seiner hohen Effektivität in der antiarrhythmischen Therapie besitzt Amiodaron ein breites Spektrum an möglichen unerwünschten Wirkungen, wobei auch die Schilddrüse auf unterschiedliche Art und Weise betroffen sein kann. Einerseits enthält Amiodaron verhältnismäßig große Mengen an Jod (75 mg Jod pro $200 \mathrm{mg}$ Amiodaron), andererseits kann durch intrinsische Effekte die Umwandlung von Thyroxin (T4) in Trijodthyronin (T3) sowie die Bindung von T3 an den nukleären Rezeptor behindert werden [1]. Zudem wird angenommen, dass es durch diverse toxische Wirkungen auf die Follikelepithelzellen $\mathrm{zu}$ einer destruktiven Thyreoiditis kommen kann [1]. Entsprechend entwickeln ca. 15-20\% der mit Amiodaron behandelten PatientInnen entweder eine Amiodaron-induzierte Hypothyreose (AIH) oder eine Amiodaron-induzierte Thyreotoxikose (AIT) [2]. Prinzipiell wird empfohlen, dass vor Beginn einer Therapie mit Amiodaron eine Bestimmung von Thyroideastimulierendem Hormon (TSH), freiem Thyroxin (fT4), freiem Trijodthyronin (fT3) sowie der Thyreoperoxidase(TPO)-Antikörper erfolgt. Dadurch sollen Personen mit bereits bestehender Schilddrüsenfunktionsstörung bzw. jene, die ein höheres Risiko für die Entwicklung einer solchen besitzen, identifiziert werden [1]. Auch anschließend sollten während der laufenden Therapie zumindest TSH und fT4 alle 3-6 Monate kontrolliert werden und PatientInnen auf das Erkennen möglicher Symptome einer Hyper- oder Hypothyreose geschult werden [1]. Die Einnahme von Amiodaron führt auch bei Personen ohne Schilddrüsenfunktionsstörungen $\mathrm{zu}$ akuten und chronischen Veränderungen der Schilddrüsenfunktionsparameter: Aufgrund des hohen Jodgehaltes von Amiodaron und des Wolff-ChaikoffEffekts kommt es zu einer reduzierten Produktion von Schilddrüsenhormonen und somit zu einem Anstieg von TSH. Durch eine Hemmung der hepatischen Typ-1-Jodothyronin-Dejodase steigt der Serumspiegel von fT4, während der Spiegel von fT3 sinkt. Wenn die Therapie mit Amiodaron über mehr als drei Monate fortgesetzt wird, kommt es zu einer zunehmenden Normalisierung dieser Veränderungen: TSH liegt meist im Normbereich, während sich fT3 oft im unteren Normbereich befindet und fT4 grenzwertig hoch oder leicht erhöht ist [1-3]. Entsprechend werden PatientInnen zu diesem Zeitpunkt bei normalem TSH und leicht erhöhtem fT4 als euthyreot angesehen [1]. Der vorliegende Artikel wurde vor allem in Anlehnung an die Guidelines der Europäischen Schilddrüsengesellschaft verfasst [2].

\section{Amiodaron-induzierte Hypothyreose (AIH)}

Die AIH tritt häufig bei PatientInnen mit vorbestehenden Autoimmunerkrankungen der Schilddrüse auf [4]. Sie weist außerdem eine höhere Prävalenz bei Frauen sowie in Jodmangelgebieten auf [5]. In allen Fällen einer manifesten Hypothyreose wird eine Substitutionstherapie mit Levothyroxin empfohlen. Die Diagnose wird anhand eines erhöhten TSH bei gleichzeitig erniedrigtem fT4 gestellt (das fT3 ist unter Einnahme von Amiodaron auch bei klinischer Euthyreose oft erniedrigt oder im niedrigen Normbereich, siehe oben) [2]. Eine latente Hypothyreose muss nicht in jedem Fall behandelt werden, insbesondere bei älteren PatientInnen, geringen TSH-Erhöhungen und asymptomatischen Personen kann zunächst eine regelmäßige Kontrolle der Schilddrüsenparameter erfolgen und mit der Levothyroxintherapie zugewartet werden $[1,2]$. Innerhalb der ersten Monate der Amiodarontherapie muss außerdem bedacht werden, dass das TSH durch den WolffChaikoff-Effekt in direkter Konsequenz des Jodüberschusses erhöht ist und somit nicht unkritisch zur Diagnose einer latenten Hypothyreose herangezogen werden sollte [1]. Aufgrund einer AIH und der einfachen und unkomplizierten Therapie ist ein Absetzen von Amiodaron nicht zwingend notwendig [2]. Eine klare Assoziation zwischen der täglichen oder kumulativen Dosis von Amiodaron und dem Auftreten einer AIH besteht nicht $[5,6]$.

\section{Amiodaron-induzierte Thyreotoxikose (AIT)}

Bei der AIT werden üblicherweise hinsichtlich der Entstehung und des zugrunde liegenden Pathomechanismus zwei Subtypen unterschieden. Eine Typ1-AIT (AIT 1) ist die Konsequenz des Jodexzesses durch die Amiodarontherapie, der bei PatientInnen mit Morbus Basedow oder Schilddrüsenautonomie zu einer unkontrollierten Synthese von Schilddrüsenhormonen führt [4]. Die Typ-2-AIT (AIT 2) ist eine durch 


\begin{tabular}{|c|c|c|}
\hline & AIT 1 & AIT 2 \\
\hline Vorbekannte Schilddrüsenerkrankung & $\begin{array}{l}\text { Häufiger (Morbus Basedow oder Schilddrüsenauto- } \\
\text { nomie) }\end{array}$ & Nein bzw. selten \\
\hline Beginn & Kurz nach Therapiebeginn & $\begin{array}{l}\text { Häufig spät (auch Jahre nach Therapiebe- } \\
\text { ginn) }\end{array}$ \\
\hline Schilddrüsenantikörper & Teilweise vorhanden (bei Morbus Basedow) & Fehlend \\
\hline Farb-Doppler-Sonographie & Erhöhte Durchblutung & Reduzierte Durchblutung \\
\hline Spitzenfluss in der Arteria thyreoidea superior & Hoch (oft $\geq 50 \mathrm{~cm} / \mathrm{s}$ ) & Niedrig (oft $<50 \mathrm{~cm} / \mathrm{s}$ ) \\
\hline $\begin{array}{l}\text { 24-Stunden-Radiojoduptake (in Jodmangelge- } \\
\text { bieten) }\end{array}$ & Erniedrigt, normal oder erhöht & Supprimiert \\
\hline Serum-Interleukin-6 und Thyreoglobulin & Normal oder niedrig & Erhöht \\
\hline
\end{tabular}

Amiodaron ausgelöste destruktive Thyreoiditis, bei welcher es aufgrund der Zerstörung von Schilddrüsenzellen zur Entstehung einer Thyreotoxikose kommt [7]. Dennoch ist eine Unterscheidung der beiden Typen leider nicht in allen Fällen klar zu treffen, sodass auch „Mischtypen“ vorkommen können [2].

Für die Diagnose einer AIT wird generell ein supprimiertes TSH bei gleichzeitig erhöhtem fT3 und fT4 benötigt [2]. In manchen Fällen kann jedoch fT3 im Sinne einer schweren „non-thyroidal illness" trotzdem im Normbereich liegen, während fT4 gerade am Anfang der Erkrankung auch ohne behandlungsbedürftige Thyreopathie gering erhöht sein kann $[8,9]$.

Die Unterscheidung der Subtypen der AIT stellt in vielen Fällen eine klinische Herausforderung dar, insbesondere, da kein einzelner klinischer oder laborchemischer Parameter zur sicheren Unterscheidung existiert [2]. Dennoch ist eine Differenzierung von großer klinischer Bedeutung, da die Erstlinientherapie je nach Subklassifikation variiert. Somit muss der weitere Behandlungsplan immer unter Berücksichtigung verschiedener Untersuchungsbefunde und in deren Zusammenschau adaptiert werden, wobei laborchemische, sonographische und nuklearmedizinische Verfahren zum Einsatz kommen [7].

Hinsichtlich laborchemischer Untersuchungen kann die Erhebung bestimmter Parameter in der Subtypisierung einer AIT hilfreich sein, dennoch kann kein Befund allein das Vorliegen eines bestimmten Subtyps ausschließen oder bestätigen. Schilddrüsenantikör- per (und TSH-Rezeptor-Antikörper im Speziellen) sind häufiger bei AIT 1 als bei AIT 2 positiv, insbesondere wenn ein Morbus Basedow als Prädisposition vorliegt [1]. Darüber hinaus konnten erhöhte Serumspiegel von Thyreoglobulin und Interleukin-6 mit dem Vorliegen einer AIT 2 in Verbindung gebracht werden, wobei auch deren Sensitivität und Spezifität als alleiniger Parameter nicht ausreichend für eine verlässliche Differenzierung ist [1]. Durch eine Schilddrüsensonographie lassen sich schnell und einfach das Schilddrüsenvolumen, die Echogenität des Parenchyms sowie das mögliche Vorliegen von Schilddrüsenknoten evaluieren. Während das sonographische Bild einer Autoimmunthyreopathie oder einer Knotenstruma häufiger bei AIT 1 auffällt, können auch bei AIT 2 morphologische Schilddrüsenveränderungen auftreten [1, 2]. Von größerer diagnostischer Wertigkeit dürfte die Farb-Doppler-Sonographie zur Beurteilung der Schilddrüsendurchblutung sein $[10,11]$. Dahinter steht die Überlegung, dass es sich bei der AIT 1 um eine Form der Thyreotoxikose infolge einer exzessiven Schilddrüsenhormonproduktion wie bei Morbus Basedow oder einer Schilddrüsenautonomie handelt, welche mit einem erhöhten Blutfluss in der Schilddrüse einhergehen würde. Hingegen wäre dies bei einer destruktiven Thyreoiditis wie einer AIT 2 nicht zu erwarten [1]. Auch eine Messung der systolischen Spitzenflussgeschwindigkeit in der Arteria thyreoidea superior kann sich für die Subtypendifferenzierung als hilfreich herausstellen [12]. In Jodmangelgebieten ist der 24-Stunden-
Raidiojoduptake bei AIT 2 meistens nicht vorhanden, wohingegen der Uptake bei AIT 1 niedrig, normal oder sogar hoch sein kann. In Gebieten mit ausreichender Jodversorgung ist eine Messung des 24-Stunden-Raidiojoduptakes nicht zielführend, da ein fehlender Uptake bei allen PatientInnen vorkommen kann [2, 13]. - Tab. 1 fasst mögliche Untersuchungsbefunde und Unterscheide der AIT-Subtypen zusammen.

Die Behandlung der AIT hängt maßgeblich von der Subtypisierung ab. Aufgrund des pathophysiologischen Mechanismus und der exzessiven Schilddrüsenhormonproduktion stellt eine thyreostatische Therapie mit Thionamiden bei AIT 1 (oder einem hinreichenden Verdacht auf eine eindeutige AIT 1) die Erstlinientherapie der Wahl dar. Oft werden sehr hohe Dosen benötigt (bis $40 \mathrm{mg}$ Methimazol/Thiamazol täglich bzw. äquivalente Dosen von Propylthiouracil), außerdem ist häufig auch die Therapiedauer bis zum Erreichen einer Euthyreose verhältnismäßig lang [2]. Um die Aufnahme von Jod in die Schilddrüse zu blockieren und die Sensitivität der Schilddrüse gegenüber Thionamiden zu verbessern, kann ergänzend eine Therapie mit Natriumperchlorat eingeleitet werden [4], die Gabe von Natriumperchlorat sollte jedoch nicht länger als über einen beschränkten Zeitraum von 4-6 Wochen erfolgen [2]. Die Behandlung mit Thionamiden kann bis zum Erreichen einer Euthyreose fortgeführt werden, sofern die Schwere der kardiologischen Grunderkrankung keine notfallmäßige definitive Therapie (d.h. eine Thyreoidektomie) notwendig 
macht. Nach Erreichen einer Euthyreose wird bei AIT 1 eine definitive Therapie, d. h. eine Thyreoidektomie bzw. eine Radioiodtherapie, empfohlen (wobei mit einer Radioiodtherapie ca. 6-12 Monate nach Absetzen von Amiodaron zugewartet werden muss) [2].

Im Gegensatz zur AIT 1 steht bei der AIT 2 die Zerstörung von Schilddrüsengewebe im Sinne einer destruktiven Thyreoiditis im Zentrum des Pathomechanismus. Da die Synthese der Schilddrüsenhormone nicht wie bei der AIT 1 exzessiv gesteigert ist, kann durch Thyreostatika keine Verbesserung der Hyperthyreose erzielt werden [4]. Obwohl die AIT 2 manchmal einen sehr prolongierten Verlauf zeigt, ist sie prinzipiell eine selbstlimitierende Erkrankung, sodass milde Fälle keine zwingende Therapie erfordern [7]. Da schwerere Hyperthyreosen jedoch die kardiologische Grunderkrankung verschlechtern können, erfordern diese Situationen eine medikamentöse Intervention. Obwohl die Empfehlung auf wenigen Studien mit eingeschränkter Datenlage beruht, stellen Glukokortikoide derzeit die Therapie der Wahl bei AIT 2 dar [2]. Üblicherweise wird eine Stardosis von $30 \mathrm{mg}$ Prednison (oder äquivalente Dosen eines anderen Glukokortikoids) empfohlen, wobei sich die weitere Dosis bzw. ein Tapering an der klinischen und laborchemischen Schilddrüsenfunktion orientieren sollte [2,9]. PatientInnen mit einer reinen AIT2 zeigen typischerweise ein sehr rasches Ansprechen auf die Glukokortikoidtherapie, sodass bei ca. $50 \%$ innerhalb von nur vier Wochen eine Euthyreose wiederhergestellt werden kann [14]. Dennoch muss angemerkt werden, dass auch bei AIT 2 ein prolongierter Verlauf möglich ist, der teilweise eine Glukokortikoidtherapie über viele Monate notwendig macht [14].

Eine der größten Schwierigkeiten bei der Behandlung von Amiodaron-assoziierten Thyreopathien stellt die Therapie von Mischtypen bzw. undefinierten Typen der AIT dar. Bei diesen Formen der AIT spielen vermutlich sowohl die pathogenetischen Mechanismen der AIT 1 (exzessive Schilddrüsenhormonsynthese) als auch die der AIT 2 (Freisetzung von Schilddrüsenhormonen durch Zer-

J. Klin. Endokrinol. Stoffw. 2020 · 13:119-123 https://doi.org/10.1007/s41969-020-00109-6 (c) Der/die Autor(en) 2020

\section{Trummer $\cdot$ V. Theiler-Schwetz $\cdot$ S. Pilz Amiodaron-induzierte Thyreopathien}

\section{Zusammenfassung}

Amiodaron ist ein Klasse III-Antiarrhythmikum mit einem breiten Spektrum von Wirkungen auf die Schilddrüse, wodurch sowohl eine Schilddrüsenunterfunktion als auch eine Schilddrüsenüberfunktion entstehen kann. Dies ist zum einen durch den sehr hohen Jodgehalt von Amiodaron und zum anderen durch das mögliche Auslösen einer destruktiven Thyreoiditis zu erklären. Eine Amiodaron-induzierte Hypothyreose (AIH) wird wie auch sonst üblich mit Levothyroxin behandelt, ein Absetzen von Amiodaron ist deshalb nicht notwendig. Bei der Amiodaroninduzierten Thyreotoxikose (AIT) werden zwei Formen unterschieden: Die AIT 1 entsteht durch den Jodexzess bei Amiodarontherapie auf dem Boden eines Morbus Basedow oder einer Schilddrüsenautonomie, die AIT 2 stellt eine destruktive Thyreoiditis dar. Diese Unterscheidung ist bei der Diagnostik von großer Bedeutung, da je nach Subtyp eine unterschiedliche Erstlinientherapie begonnen wird. Die AIT 1 wird zunächst mit Thionamiden ( \pm Natriumperchlorat) behandelt, PatientInnen mit AIT 2 erhalten zu Beginn Glukokortikoide. Falls eine genaue Einteilung in einen der beiden Subtypen nicht möglich ist (Mischtypen/undefinierte Typen), kann zunächst das Ansprechen auf Thionamide getestet werden oder von Beginn an eine Kombinationstherapie mit Glukokortikoiden erfolgen. Amiodaron muss insbesondere bei milden Fällen einer AIT 2 nicht abgesetzt werden, bei AIT 1 sollte ein Absetzen erwogen werden. In Notfällen sowie bei kritisch kranken PatientInnen kann auch eine Thyreoidektomie erfolgen.

\section{Schlüsselwörter}

Amiodaron - Hyperthyreose $\cdot$ Hypothyreose . Glukokortikoide - Thionamide

\section{Amiodarone-induced thyroid disorders}

\section{Abstract}

Amiodarone, a class III antiarrhythmic drug, has several adverse effects on the thyroid that may lead to both hypothyroidism and hyperthyroidism. On one hand, this can be attributed to its high content of iodine, and on the other hand, amiodarone may cause destructive thyroiditis. Amiodaroneinduced hypothyroidism (AIH) is treated with levothyroxine replacement therapy and does not necessitate the discontinuation of amiodarone. Amiodarone-induced thyrotoxicosis (AIT) can be categorized into two subtypes: AIT 1 is the consequence of iodine overload in underlying Graves' disease or thyroid autonomy, while AIT 2 represents a form of destructive thyroiditis. Since the first line therapy differs significantly between the two subtypes, a thorough diagnostic workup is paramount. AIT 1 is primarily treated with thionamides ( \pm sodium perchlorate), whereas the initial therapy of choice for AIT 2 is glucocorticoids. In cases where a definitive distinction between the subtypes cannot be made (mixed types/indeterminate types) it is recommended to primarily initiate a therapeutic trial with thionamides or to start a combination therapy with glucocorticoids. While amiodarone does not need to be discontinued in most, especially mild cases of AIT 2, termination of treatment should be considered in AIT 1. In emergency situations and in patients with high cardiovascular risk, thyroidectomy is a possible alternative if immediate treatment is deemed necessary.

\section{Keywords}

Amiodarone · Hyperthyroidism · Hypothyroidism · Glucocorticoids · Thionamides störung von Schilddrüsengewebe) eine Rolle [7]. Falls sich aus der Zusammenschau der diagnostischen Untersuchungen kein eindeutiger Hinweis für eine AIT 1 oder 2 ergibt, stehen zwei Therapieregime zur Wahl: Einerseits kann eine Therapie mit Thyreostatika wie bei AIT 1 (eventuell in Kombination mit Natriumperchlorat) begonnen werden.
Sollte sich darunter nach einer kurzen Zeit (ungefähr 4-6 Wochen) keine Verbesserung der Hyperthyreose zeigen, wird vom parallelen Vorliegen einer destruktiven Thyreoiditis ausgegangen und eine zusätzliche Glukokortikoidtherapie begonnen [2]. Andererseits sieht ein anderes Therapieregime vor, gleich $\mathrm{zu}$ Beginn eine Kombination aus Thionami- 
den und Glukokortikoiden einzuleiten, um eine mögliche Mischform gleich von Beginn an zu therapieren [15]. Der Nachteil dieser Vorgehensweise liegt darin, dass PatientInnen mit teilweise schweren kardialen Grunderkrankungen einer möglicherweise überflüssigen Glukokortikoidtherapie ausgesetzt werden [2].

Ob die Therapie mit Amiodaron bei einer AIT abgesetzt werden muss, muss immer im Konsens zwischen Endokrinologie und Kardiologie beschlossen werden und bedarf einer individualisierten Entscheidungsfindung. Generell muss bedacht werden, dass die Effekte von Amiodaron aufgrund seiner langen Halbwertszeit noch Monate nach Absetzen bestehen bleiben, sodass ein Therapieabbruch möglicherweise nicht zu einer unmittelbaren Normalisierung der Schilddrüsenfunktion führt [4]. Im Allgemeinen sollte Amiodaron natürlich nicht bei kritisch kranken PatientInnen abgesetzt werden, die dadurch dem Risiko möglicherweise lebensbedrohlicher kardialer Arrhythmien/Erkrankungen ausgesetzt werden. Auch bei einer AIT 2 kann - da die Erkrankung oft selbstlimitierend verläuft die Amiodarontherapie meistens fortgeführt werden [2]. Bei AIT 1 sollte hingegen ein Absetzen in Erwägung gezogen werden, falls der Benefit der Amiodarontherapie in der kardiologischen Behandlung nicht deutlich überwiegt. Diese Entscheidung sollte aber jedenfalls immer zusammen mit der/dem behandelnden KardiologIn getroffen werden [16].

Da eine Thyreotoxikose auch bei primär asymptomatischen PatientInnen zu einer Dekompensation der kardialen Grunderkrankung führen kann, sollte jeder Fall einer AIT als potenziell bedrohlich eingestuft werden und eine Euthyreose so schnell wie möglich angestrebt werden. In speziellen, besonders gefährdeten Patientengruppen kann es daher notwendig werden, eine totale Thyreoidektomie als Notfallmaßnahme durchzuführen. Dazu gehören Personen mit einem raschen Rückgang der kardialen Funktion nach Eintritt einer AIT oder PatientInnen mit schweren zugrunde liegenden Herzerkrankungen (z.B. maligne Arrhythmien) [2]. Diesbezüglich zeigte sich auch in einer aktu- ellen Studie [17] ein möglicher Benefit einer Thyreoidektomie bei PatientInnen mit höhergradig eingeschränkter Linksventrikelfunktion im Vergleich zur konventionellen medikamentösen Therapie. Eine akute Thyreoidektomie ist auch eine Therapieoption bei Fällen von therapieresistenter AIT, welche teilweise auch unter manifester Hyperthyreose durchgeführt werden muss [18]. Die Entscheidung zur operativen Sanierung sollte jedenfalls immer interdisziplinär zwischen Endokrinologie, Kardiologie, Anästhesie und Chirurgie getroffen werden [2].

Abschließend möchten wir darauf hinweisen, dass sich bei der Therapie eines Vorhofflimmerns bei gleichzeitiger manifester Hyperthyreose, z. B. bei Morbus Basedow oder Schilddrüsenautonomie, manchmal die Frage stellt, $\mathrm{ob}$ in solchen Situationen eine Therapie mit Amiodaron erfolgen darf. Prinzipiell sollte Amiodaron in solchen Situationen natürlich nicht die Therapie der ersten Wahl darstellen, kann aber in Fällen von anderwärtig nicht ausreichend kontrollierbarem Vorhofflimmern eingesetzt werden. Wichtig ist dabei jedoch immer die begleitende Gabe einer thyreostatischen Therapie sowie von Natriumperchlorat [19, 20].

\section{Korrespondenzadresse}

\section{PD DDr. Christian Trummer}

Klinische Abteilung für Endokrinologie und Diabetologie, Univ.-Klinik für Innere Medizin, Medizinische Universität Graz

Auenbruggerplatz 15, 8036 Graz, Österreich christian.trummer@medunigraz.at

\section{Assoz. Prof. PD Dr. Stefan Pilz, PhD}

Klinische Abteilung für Endokrinologie und Diabetologie, Univ.-Klinik für Innere Medizin, Medizinische Universität Graz

Auenbruggerplatz 15, 8036 Graz, Österreich stefan.pilz@chello.at

Funding. Open access funding provided by Medical University of Graz.

\section{Einhaltung ethischer Richtlinien}

Interessenkonflikt. C. Trummer, V. Theiler-Schwetz und S. Pilz geben an, dass kein Interessenkonflikt besteht.
Für diesen Beitrag wurden von den Autoren keine Studien an Menschen oder Tieren durchgeführt. Für die aufgeführten Studien gelten die jeweils dort angegebenen ethischen Richtlinien.

Open Access. Dieser Artikel wird unter der Creative Commons Namensnennung 4.0 International Lizenz veröffentlicht, welche die Nutzung, Vervielfältigung, Bearbeitung, Verbreitung und Wiedergabe in jeglichem Medium und Format erlaubt, sofern Sie den/die ursprünglichen Autor(en) und die Quelle ordnungsgemäß nennen, einen Link zur Creative Commons Lizenz beifügen und angeben, ob Änderungen vorgenommen wurden.

Die in diesem Artikel enthaltenen Bilder und sonstiges Drittmaterial unterliegen ebenfalls der genannten Creative Commons Lizenz, sofern sich aus der Abbildungslegende nichts anderes ergibt. Sofern das betreffende Material nicht unter der genannten Creative Commons Lizenz steht und die betreffende Handlung nicht nach gesetzlichen Vorschriften erlaubt ist, ist für die oben aufgeführten Weiterverwendungen des Materials die Einwilligung des jeweiligen Rechteinhabers einzuholen.

Weitere Details zur Lizenz entnehmen Sie bitte der Lizenzinformation auf http://creativecommons.org/ licenses/by/4.0/deed.de.

\section{Literatur}

1. Basaria S, Cooper DS (2005) Amiodarone and the thyroid. Am JMed 118:706-714

2. Bartalena L, Bogazzi F, Chiovato L, HubalewskaDydejczyk A, Links TP, Vanderpump M (2019) 2018 European Thyroid Association (ETA) guidelines for the management of amiodarone-associated thyroid dysfunction. Eur Thyroid J 7:55-66

3. Amico JA, Richardson V, Alpert B, Klein I (1984) Clinical and chemical assessment of thyroid function during therapy with amiodarone. Arch Intern Med 144:487-490

4. Martino E, Bartalena L, Bogazzi F, Braverman LE (2001) The effects of amiodarone on the thyroid. Enodcr Rev 22:240-254

5. Zhong B, Wang Y, Zhang G, Wang Z (2016) Environmental iodine content, female sex and age are associated with new-onset amiodaroneinduced hypothyroidism: a systemic review and meta-analysis of adverse reactions of amiodarone on the thyroid. Cardiology 134:366-371

6. Bogazzi F, TomistiL, BartalenaL,Aghini-LombardiF, Martino E (2012) Amiodarone and the thyroid: a 2012 update. J Endocrinol Invest 35:340-348

7. Bogazzi F, Bartalena L, Martino E (2010) Approach to the patient with amiodarone-induced thyrotoxicosis. JClin Endocrinol Metab 95:2529-2535

8. Balzano S, Sau F, Bartalena L, Ruscazio M, Balestrieri A, Cherchi A, Martino E (1987) Diagnosis of amiodarone-iodine-induced thyrotoxicosis (AIIT) associated with severe non-thyroidal illness. JEndocrinol Invest 10:589-591

9. Han TS, Williams GR, Vanderpump MPJ (2009) Benzofuran derivatives and the thyroid. Clin Endocrinol (Oxf) 70:2-13

10. Bogazzi F, Bartalena L, Brogioni S, Mazzeo S, Vitti $P$, Burelli A, Bartolozzi C, Martino E (1997) Color flow Doppler sonography rapidly differentiates type and type II amiodarone-induced thyrotoxicosis. Thyroid 7:541-545

11. Theodoraki A, Vanderpump MPJ (2016) Thyrotoxicosis associated with the use of amiodarone: the 
utility of ultrasound in patient management. Clin Endocrinol (Oxf) 84:172-176

12. Zhao $X$, Chen $L$, Li L, Wang $Y$, Wang $Y$, Zhou L, Zeng F, Li Y, Hu R, Liu H (2012) Peak systolic velocity of superior thyroid artery for the differential diagnosis of thyrotoxicosis. Plos One 7:e50051

13. Bartalena L, Bogazzi F, Braverman LE, Martino E (2001) Effects of amiodarone administration during pregnancy on neonatal thyroid function and subsequent neurodevelopment. J Endocrinol Invest 24:116-130

14. Bogazzi F, Bartalena L, Tomisti L, Rossi G, Tanda ML Dell'Unto E, Aghini-Lombardi F, Martino E (2007) Glucocorticoid response in amiodaroneinduced thyrotoxicosis resulting from destructive thyroiditis is predicted by thyroid volume and serum free thyroid hormone concentrations. J Clin Endocrinol Metab 92:556-562

15. Vanderpump MPJ (2009) Thyroid gland: use of glucocorticoids in amiodaron-induced thyrotoxicosis. Nat Rev Endocrinol 5:650-651

16. Tanda ML, Piantanida E, Lai A, Liparulo L, Sassi L, Bogazzi F, Wiersinga WM, Braverman LE, MartinoE, Bartalena $L$ (2008) Diagnosis and management of amiodarone-induced thyrotoxicosis: similarities and differences between North America and European thyroidologists. Clin Endocrinol (Oxf) 69:812-818

17. Capellani D, Papini P, Pingitore A, Tomisti L, Mantuano M, Di Certo AM, Manetti L, Marconcini G, Scattina I, Urbani C, Morganti R, Marcocci C, Materazzi G, lervasi G, MartinoE, Bartalena L, Bogazzi F (2020) Comparison between total thyroidectomy and medical therapy for amiodarone-induced thyrotoxicosis. JClin Endocrinol Metab 105:dgz041

18. Kotwal A, Clark J, Lyden M, McKenzie T, Thompson G, Stan MN (2018) Thyroidectomy for amiodarone-induced thyrotoxicosis: Mayo Clinic experience. J Endocr Soc 2:1226-1235

19. Parmar MS (2005) Thyrotoxic atrial fibrillation. MedGenMed 7:74

20. Unger J, Lambert $\mathrm{M}$, Jonckheer $\mathrm{MH}$, Denayer $\mathrm{P}$ (1993) Amiodarone and the thyroid: pharmacological, toxic and therapeutic effects. J Intern Med 233:435-443

Hinweis des Verlags. Der Verlag bleibt in Hinblick auf geografische Zuordnungen und Gebietsbezeichnungen in veröffentlichten Karten und Institutsadressen neutral.

\section{Neuer Antikörper könnte Eisenstoffwechsel bei Dialyse- PatientInnen verbessern}

Ein Team der Medizin Uni Innsbruck hat einen Antikörper für die Therapie der chronischen Blutarmut bei Dialyse-PatientInnen mitentwickelt. Der Mehrwert des neuen Ansatzes liegt in der geringer benötigten EPO-Dosis und der damit einhergehenden Minimierung des kardioavaskulären Risikos, dem NierenpatientInnen ausgesetzt sind. Nach den vielversprechenden Ergebnissen im Tiermodell, soll der Antikörper bald in die klinische Erprobung gehen.

Die chronische Blutarmut ist ein häufiges Begleitproblem bei Dialyse-PatientInnen. Neben dem funktionellen Eisenmangel, kommt es durch die geschädigte Niere zu einer geringen Produktion des für die Blutbildung wichtigsten Hormons, Erythropoetin (EPO). Die EPO-Gabe stellt daher einen Grundpfeiler in der Therapie der Anämie dar. Dennoch bleibt die Bildung von Hämoglobin bei Dialyse-PatientientInnen aufgrund einer Resistenzentwicklung gegen EPO eingeschränkt. Auch wird das Therapeutikum EPO mit einer erhöhten kardiovaskulären Mortalität in Verbindung gebracht. „Dies stellt für die PatientInnen ein zusätzliches Dilemma dar, da eine Niereninsuffizienz an sich schon mit einem erhöhten kardiovaskulären Risiko vergesellschaftet ist", betont lgor Theurl, der gemeinsam mit Günter Weiss seit vielen Jahren zur Anämie chronischer Erkrankungen forscht.

Antikörper hemmt Eisenregulator Hepcidin

In Zusammenarbeit mit der Pharmafirma Kymab, Cambridge, testete das Team den neuen humanen monoklonalen Antikörper KY1070. In Innsbruck konnte dessen Wirksamkeit in einem Tiermodell aufgezeigt werden. „In Tieren, die nur unzureichend auf EPO angesprochen haben, war es durch Kombination mit dem Antikörper möglich, einen ausreichenden HämoglobinAnstieg zu erreichen. Außerdem reichte eine geringere EPO-Dosis aus, um die Anämie zu bekämpfen, was in Anbetracht der Nebenwirkungen von EPO eine wichtige Grundlage zum Einsatz bei DialysePatientInnen darstellt", so Erstautorin Verena Petzer.

Potenzial für Mono- und Kombinationstherapie

Das therapeutische Potenzial des neuen Antikörpers lässt sich als Monotherapie wie auch in Kombination mit EPO verwerten. Der Antikörper beeinflusst die Signalweiterleitung zur Bildung von Hepcidin, einem zentralen Regulator für den Eisenstoffwechsel. Als Monotherapie kommt es zu einer langandauernden Hemmung der Hepcidinproduktion und so zu einer hohen Eisenverfügbarkeit, die für die Bildung von roten Blutkörperchen essentiell ist. „Die Effekte der Kombinationstherapie sind multifaktoriell. Der Vorteil dieser Behandlung liegt in der Reduktion der EPODosis, sodass das kardiovaskuläre Risiko nicht zusätzlich erhöht wird und gleichzeitig eine ausreichende Eisenverfügbarkeit sichergestellt wird. Auch konnten wir zeigen, dass durch die Hemmung von Hepcidin die Expression des Eisentransportproteins Ferroportin auf den blutbildenden Zellen im Knochenmark gesteigert wird und gemeinsam mit EPO zu einer effektiveren Blutbildung führt" so Igor Theurl.

Anämieforschung in Innsbruck Günter Weiss und Igor Theurl konnten in den letzten Jahren bereits wichtige pathophysiologische Mechanismen, die zur Ausbildung der ACD führen, aufklären - als wesentlicher Mechanismus liegt der $A C D$ eine vermehrte Speicherung von Eisen in den Fresszellen des Immunsystems zugrunde. Dies führt zu einer verminderten Verfügbarkeit des Eisens für die Blutbildung, wo das Eisen für die Herstellung des roten Blutfarbstoffes Hämoglobin benötigt wird die Folge: Anämie.

Original publikation: A fully human anti-BMP6 antibody reduces the need for erythropoietin in rodent models of the Anemia of Chronic Disease., V. Petzer et al., Blood 27;136(9):10801090 (2020).

Quelle: Doris Heidegger, Medizinische Universität Innsbruck 